\title{
Dust Absorption and Emission in Galaxies at High and Low Redshifts
}

\section{S. Michael Fall}

Space Telescope Science Institute, 3700 San Martin Drive, Baltimore, $M D$ 21218, USA

\begin{abstract}
This article reviews three related topics: the extragalactic background light and its sources, evolution models for the dust absorption and emission in galaxies, and empirical constraints on these transfer processes in nearby starburst galaxies. It is intended that the material presented here will serve as an introduction to this Joint Discussion.
\end{abstract}

\section{Extragalactic Background Light}

A good place to begin our discussion of the effects of dust on the radiation from galaxies is with the extragalactic background light (EBL). We denote the intensity of this isotropic radiation per unit frequency $\nu$ by $J_{\nu}$ and the integral of this over all $\nu$, the bolometric intensity, by $J_{\text {bol }}$. By definition, $J_{\nu}$ excludes foreground radiation from the Milky Way and other nearby galaxies and the cosmic microwave background radiation from the Big Bang, but it includes the radiation from everything in between. A few years ago, we had only rough estimates and upper or lower limits on $J_{\nu}$ at most wavelengths, but this situation has improved dramatically, thanks largely to $H S T$ and $C O B E$. We now know $J_{\nu}$ to an accuracy of a factor of two or better over four decades in wavelength, from about 0.2 to $2000 \mu \mathrm{m}$. This is shown in Figure 1 below.

Two interesting results follow directly from Figure 1 . First, emission by dust is important. There is at least as much energy in the long-wavelength hump of the EBL spectrum $(\lambda \gtrsim 10 \mu \mathrm{m})$ as in the short-wavelength hump $(\lambda \lesssim 10 \mu \mathrm{m})$. Second, integrations under the smooth curves in Figure 1 give

$$
J_{\mathrm{bol}} \approx 50 \mathrm{nWm}^{-2} \mathrm{sr}^{-1} \text {. }
$$

This is a summary record of the bolometric emissivity $E_{\text {bol }}$ (power radiated per unit comoving volume) integrated over the age of Universe $t_{0}$ :

$$
J_{\mathrm{bol}}=\frac{c}{4 \pi} \int_{0}^{t_{0}} \frac{E_{\mathrm{bol}}}{1+z} d t .
$$

Equations (1) and (2) provide valuable constraints on possible sources of the $E B L$, independent of the complex radiative transfer within galaxies.

The most promising sources of the EBL are stars (nuclear energy) and AGN (accretion onto black holes, gravitational energy). Equation (2) enables us to express these contributions to the EBL in terms of the present comoving densities of stars and black holes (normalized by the critical density). Recent 


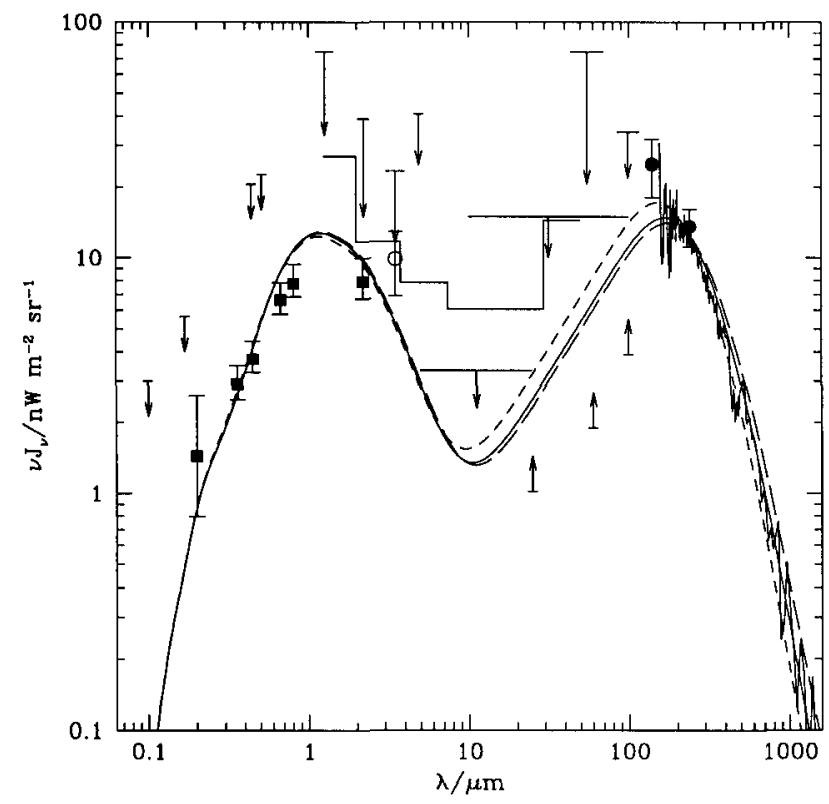

Figure 1. Spectrum of the extragalactic background light, $\nu J_{\nu}$ versus $\lambda$. See Pei, Fall, \& Hauser (1999) for references to the observations. The curves are from the global evolution models in the same paper.

estimates give $\Omega_{\mathrm{S}} \approx 4 \times 10^{-3}$ and $\Omega_{\mathrm{BH}} \approx 2 \times 10^{-5}$ for $H_{0}=70 \mathrm{kms}^{-1} \mathrm{Mpc}^{-1}$. For stars, we obtain from population synthesis models

$$
J_{\mathrm{S}} \approx 50 f\left(\Omega_{\mathrm{S}} / 4 \times 10^{-3}\right) \mathrm{nWm}^{-2} \mathrm{sr}^{-1},
$$

where $f \approx 1$ depends on the history of star formation and the initial mass function. For AGN, we obtain

$$
J_{\mathrm{AGN}} \approx 6(\epsilon / 0.05)\left[3 /\left(1+z_{\mathrm{acc}}\right)\right]\left(\Omega_{\mathrm{BH}} / 2 \times 10^{-5}\right) \mathrm{nWm}^{-2} \mathrm{sr}^{-1},
$$

where $\epsilon$ is the efficiency of energy conversion (gravitational to radiative) and $z_{\text {acc }}$ is the effective redshift of accretion. According to these estimates, which have substantial uncertainties, stars alone could produce the entire EBL, while AGN may make a significant but smaller contribution. Similar conclusions have been reached by Fabian \& Iwasawa (1999) and Madau \& Pozzetti (2000).

\section{Evolution Models}

Several methods have been developed to interpret or predict the appearance of galaxies at different redshifts and their contribution to the cosmic emissivity and EBL. In many cases, the absorption and reradiation of starlight by dust is governed by an effective optical $\operatorname{depth} \tau_{\text {dust }}$ that is related to the metallicity, column density, and mass of the interstellar medium, $Z_{\mathrm{ISM}}, N_{\mathrm{ISM}}, M_{\mathrm{ISM}}$, and 
the size of a galaxy $R_{\text {gal }}$ by expressions of the form

$$
\tau_{\text {dust }} \propto Z_{\mathrm{ISM}} N_{\mathrm{ISM}} \propto Z_{\mathrm{ISM}}\left(M_{\mathrm{ISM}} / R_{\mathrm{gal}}^{2}\right) .
$$

The mass and metallicity of the ISM are then related to the prior history of star formation by the equations of galactic chemical evolution, with or without inflow or outflow terms. Most recent models can be classified into three types: "backward" evolution models, "forward" evolution models, and "global" evolution models.

In backward evolution models, the present-day population of galaxies is evolved into the past using plausible rules for the histories of star formation, gas consumption, and so forth in galaxies of different types (see Franceschini et al. 1994 and references therein). Interactions and merging of galaxies are usually ignored in this approach. In forward evolution models, initial distributions of dark matter and baryons are evolved into the future using plausible rules for the merging of halos, inflow and outflow of gas, and so forth (see Granato et al. 2000 and references therein). These effects are usually treated by semianalytical techniques in the framework of the cold dark matter cosmology. Since this approach includes many different processes, it requires a large number of assumptions and parameters.

Global evolution models are based on the combined or average properties of galaxies (see Pei, Fall, \& Hauser 1999 and references therein). The quantities of interest here are the mean comoving densities of stars, ISM, metals, and dust $\left(\Omega_{\mathrm{S}}, \Omega_{\mathrm{ISM}}, \Omega_{\mathrm{M}}\right.$, and $\left.\Omega_{\mathrm{D}}\right)$ and the mean comoving emissivities of stars and dust $\left(E_{\mathrm{S} \nu}\right.$ and $\left.E_{\mathrm{D} \nu}\right)$. These quantities are governed by simple, conservationtype equations, analogous to the equations of galactic chemical evolution. The input to and output from the global evolution models include both emission and absorption-line observations, i.e., information about both the stellar and interstellar contents of galaxies. The advantages of this approach are that it is based directly on global quantities, requires relatively few assumptions and parameters, and relates the emission histories of galaxies to their absorption histories. A limitation of the global evolution models is that they do not describe the properties of individual galaxies and hence cannot be compared with luminosity functions and number counts. Moreover, the accuracy that is currently achievable is limited by small-number statistics in the existing samples of damped Ly $\alpha$ absorbers.

\section{Nearby Starburst Galaxies}

To determine the rate of stellar nucleosynthesis or black hole accretion in a galaxy, one must measure its bolometric luminosity. However, this is not currently practicable for most of the apparently faint galaxies at high redshifts. Most observations of high-redshift galaxies are restricted to rest-frame UV and optical wavelengths, which miss the light that is absorbed and reradiated by dust. Recent observations at $850 \mu \mathrm{m}$ with SCUBA help, but much of the dust emission is almost certainly at shorter wavelengths. This situation has prompted several schemes to correct the observed UV and optical fluxes to bolometric fluxes. One of the most promising of these was discovered by Meurer et al. (1995) and refined by Meurer, Heckman, \& Calzetti (1999). 
The Meurer et al. relation is based on the observed properties of UVselected, nearby starburst galaxies. These are of special interest because they may be low-redshift analogs of the high-redshift $(z \approx 3-4)$ galaxies revealed by the Lyman-break technique (Steidel et al. 1996). In the nearby sample, Meurer et al. found a relatively tight correlation between the ratio of FIR and UV fluxes, $F_{\text {dust }} / F_{1600}$, and the UV spectral slope, $\beta$. The correlation may also hold for the Lyman-break galaxies, although the observational tests for this are not strong (Adelberger \& Steidel 2000). The Meurer et al. relation is useful because, for galaxies that obey it, one can infer the FIR flux $F_{\text {dust }}$ and hence the bolometric flux from observations in the rest-frame UV $\left(F_{1600}\right.$ and $\left.\beta\right)$. In this way, Meurer el al. (1999) and Adelberger \& Steidel (2000) have estimated bolometric correction factors of about 5 between the UV-only and total star formation rates in Lyman-break galaxies at $z \approx 3-4$.

The existence of the Meurer et al. relation is closely linked to the existence of a "universal" effective absorption curve, although not quite the same as the one proposed by Calzetti, Kinney, \& Storchi-Bergmann (1994). In this case, the parameter along the sequence is the effective optical depth at some fiducial wavelength and hence the overall dust content of the galaxies. Another curious fact about the UV-selected, nearby starburst galaxies is that the apparent absorption inferred from the $\mathrm{H} \alpha / \mathrm{H} \beta$ ratio is typically twice that inferred from the UV spectral slope $\beta$ (Calzetti et al. 1994). In an effort to understand these observations, Charlot \& Fall (2000) have constructed some simple models in which stars are born in dusty clouds with finite lifetimes. As a result, young, UV-bright stars are more obscured than old, UV-faint stars. In particular, the Meurer et al. relation, and the other average spectral properties of the UVselected, nearby starburst galaxies, can be reproduced if the effective absorption is proportional to $\lambda^{-0.7}$ and is three times higher for stars younger than $10^{7} \mathrm{yr}$ than it is for older stars.

\section{References}

Adelberger, K. L., \& Steidel, C. C. 2000, ApJ, in press (astro-ph/0001126)

Calzetti, D., Kinney, A. L., \& Storchi-Bergmann, T. 1994, ApJ, 429, 582

Charlot, S., \& Fall, S. M. 2000, ApJ, 539, 718

Fabian, A. C., \& Iwasawa, K. 1999, MNRAS, 303, L34

Franceschini, A., Mazzei, P., de Zotti, G., \& Danese, L. 1994 ApJ, 427, 140

Granato, G. L., Lacey, C. G., Silva, L., Bressan, A., Baugh, C. M., Cole, S., \& Frenk, C. S. 2000, ApJ, in press (astro-ph/0001308)

Madau, P., \& Pozzetti, L. 2000, MNRAS, 312, L9

Meurer, G. R., Heckman, T. M., \& Calzetti, D. 1999, ApJ, 521, 64

Meurer, G. R., Heckman, T. M., Leitherer, C., Kinney, A., Robert, C., \& Garnett, D. R. 1995, AJ, 110, 2665

Pei, Y. C., Fall, S. M., \& Hauser, M. G. 1999, ApJ, 522, 604

Steidel, C. C., Giavalisco, M., Pettini, M., Dickinson, M., \& Adelberger, K. L. 1996, ApJ, 462, L17 